\title{
Theoretical Studies of ESR Parameters and Local Lattice Structure of the Vanadate-Lithium-Borate Glasses
}

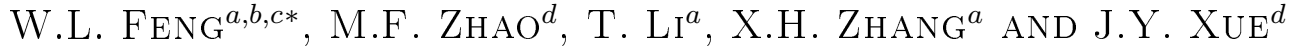 \\ ${ }^{a}$ Department of Applied Physics, Chongqing University of Technology, Chongqing 400054, China \\ ${ }^{b}$ Chongqing Key Laboratory of Time Grating Sensing \& Advanced Testing Technology, Chongqing, 400054, China \\ ${ }^{c}$ Department of Electronic Information and Automation, Chongqing University of Technology \\ Chongqing 400054, China \\ ${ }^{d}$ International Centre for Materials Physics, Chinese Academy of Sciences, Shenyang 110016, China
}

(Received October 1, 2011; revised version February 1, 2012; in final form February 4, 2012)

\begin{abstract}
Electron spin resonance spectral parameters of $\mathrm{V}^{4+}$ ions in vanadate-lithium-borate glasses have been calculated by the crystal-field theory. The theoretical results are $g_{\|}=1.940, g_{\perp}=1.983$ and $A_{\|}=-175 \times 10^{-4} \mathrm{~cm}^{-1}$, $A_{\perp}=-65 \times 10^{-4} \mathrm{~cm}^{-1}$ which are good agreement with the experimental values $\left(g_{\|}=1.939(3), g_{\perp}=1.998(3)\right.$ and $\left.A_{\|}=(170.6-176.4) \times 10^{-4} \mathrm{~cm}^{-1}, A_{\perp}=(61.3-71.4) \times 10^{-4} \mathrm{~cm}^{-1}\right)$. In addition, the bond lengths of the local lattice structure are, respectively, $R_{\|}=1.5 \AA$ and $R_{\perp}=1.95 \AA$ which have been shown to have a compressed tetrahedral geometry along the $\mathrm{C}_{4}$ axis.
\end{abstract}

PACS: $71.70 . \mathrm{Ch}, 75.10 . \mathrm{Dg}, 61.72 . \mathrm{Bb}, 76.30 . \mathrm{Fc}$

\section{Introduction}

Glasses containing transition metal and rare earth ions have attracted a great deal of attention because of their memory and photoconducting characteristics. They also find potential applications in the development of new lasers, optical amplifiers, or luminescent materials. ESR/ EPR technology can be used widely to investigate the local lattice structure information about the glassy network and to identify the point symmetry around the transition metal or rare earth ions [1-3].

$\mathrm{V}^{4+}$, as an important probe ion, can provide useful information regarding the structure of their network environment in a glass, because their ESR spectra are simple $[4,5]$. The ESR spectra of $\mathrm{V}^{4+}$ doped glasses have been studied extensively by many researchers in the systems of borate [6-9]. Among them, the ESR spectral parameters (characterized by $g$ factors $g_{\|}, g_{\perp}$ and hyperfine structure parameters $A_{\|}, A_{\perp}$ ) have been determined by Cozar et al. [9]. But there is no any theoretical analysis for these spectral data and local lattice structural parameters. In the present work, the authors bring out the theoretical results of the ESR spectral parameters and local lattice

\footnotetext{
* corresponding author; e-mail: wenlinfeng@126.com
}

structural data of $\mathrm{V}^{4+}$ ions in ternary vanadate-lithiumborate glasses. These results of calculations are in good agreement with experimental findings.

\section{Theoretical model}

\subsection{Two spin-orbit coupling parameters}

The linear combination of atomic orbitals-molecular orbital (LCAO-MO) method developed by Kivelson and Lee [10] for vanadium (vanadyl $\mathrm{VO}^{2+}$ ) complexes has been considered in present work. In addition, related studies have also shown that the contribution to ESR data from the spin-orbit (SO) coupling coefficient $\left(\zeta_{p}^{0}\right)$ of the ligand cannot be ignored when its magnitude close to or more than that $\left(\zeta_{d}^{0}\right)$ of the centre transition metal ion [11-13]. It is the case of this present work because the value of $\zeta_{p}^{0}\left(=150 \mathrm{~cm}^{-1}\right.$ [12] $)$ is close to that of the $\zeta_{d}^{0}\left(=248 \mathrm{~cm}^{-1}[14]\right)$.

\subsection{The superposition model}

According to the superposition model which was proposed by Newman et al. [15, 16], the tetragonal crystal-field parameters $D s$ and $D t$ can be expressed as

$$
D s=(4 / 7) \overline{A_{2}}\left(R_{0}\right)\left[\left(R_{0} / R_{\perp}\right)^{t_{2}}-\left(R_{0} / R_{\|}\right)^{t_{2}}\right],
$$




$$
D t=(16 / 21) \overline{A_{4}}\left(R_{0}\right)\left[\left(R_{0} / R_{\perp}\right)^{t_{4}}-\left(R_{0} / R_{\|}\right)^{t_{4}}\right],
$$

where $R_{0}$ is the reference distance which is characterized by the bond lengths $\left(R_{\|}, R_{\perp}\right)$ of the local lattice structure. $t_{2}(=3)$ and $t_{4}(=5)$ are the power-law exponents. The intrinsic parameters $\bar{A}_{k}\left(R_{0}\right)$ (subscript $k=2$ or 4 for $d^{n}$ electronic configuration) can be determined from the cubic crystal-field parameter $D q$ via the relation [17-19]:

$$
\bar{A}_{4}\left(R_{0}\right)=3 / 4 D q, \quad \bar{A}_{2}\left(R_{0}\right)=(8-12) \bar{A}_{4}\left(R_{0}\right) .
$$

We take $\bar{A}_{2}\left(R_{0}\right)=12 \bar{A}_{4}\left(R_{0}\right)$ in the calculation. The length of $\mathrm{V}-\mathrm{O}$ bond $R_{0} \approx R_{\perp} \approx 1.95 \AA$ which is corresponding to the cubic symmetry [20]. $R_{\|}$can be treated as fitting parameter. As much as we know, up to the present, the cubic crystal-field parameter $D q$ has not been reported. $D q\left(\mathrm{~F}^{-}\right)\left(\approx 2012 \mathrm{~cm}^{-1}\right)$ value of $\left(\mathrm{VF}_{6}\right)^{2-}$ cluster has been given in literature [21]. According to the chemical sequences of the optical spectra, there is $D q\left(\mathrm{~F}^{-}\right)<D q\left(\mathrm{O}^{2-}\right)$, thus, we take $D q\left(\mathrm{O}^{2-}\right) \approx$ $2150 \mathrm{~cm}^{-1}$ in the calculation and it can be regarded as reasonable.

\subsection{The high order perturbation formulae}

The electronic configuration of $\mathrm{V}^{4+}$ ion is $3 d^{1}$. The $g$ factors $g_{\|}, g_{\perp}$ and hyperfine structure parameters $A_{\|}, A_{\perp}$ of the ground state $b_{2 \mathrm{~g}}\left(\left|d_{x y}\right\rangle\right)$ can be obtained from high order perturbation methods [22],

$$
\begin{aligned}
& g_{\|}=g_{s}-\frac{8 k^{\prime} \zeta^{\prime}}{\Delta E\left(b_{1 \mathrm{~g}}\right)}-\frac{k \zeta^{2}}{\left[\Delta E\left(e_{\mathrm{g}}\right)\right]^{2}}-\frac{4 k^{\prime} \zeta \zeta^{\prime}}{\Delta E\left(e_{\mathrm{g}}\right) \Delta E\left(b_{1 \mathrm{~g}}\right)} \\
& -\frac{g_{s} \zeta^{2}}{\left[\Delta E\left(e_{\mathrm{g}}\right)\right]^{2}} \\
& g_{\perp}=g_{s}-\frac{2 k \zeta}{\Delta E\left(e_{\mathrm{g}}\right)}+\frac{k \zeta^{2}}{\left[\Delta E\left(e_{\mathrm{g}}\right)\right]^{2}}-\frac{2 k \zeta^{\prime 2}}{\Delta E\left(e_{\mathrm{g}}\right) \Delta E\left(b_{1 \mathrm{~g}}\right)} \\
& +\frac{2 k^{\prime} \zeta \zeta^{\prime}}{\Delta E\left(e_{\mathrm{g}}\right) \Delta E\left(b_{1 \mathrm{~g}}\right)}-\frac{2 g_{s} \zeta^{\prime 2}}{\left[\Delta E\left(b_{1 \mathrm{~g}}\right)\right]^{2}}-\frac{g_{s} \zeta^{2}}{2\left[\Delta E\left(e_{\mathrm{g}}\right)\right]^{2}}, \\
& A_{\|}=P\left(-\kappa-\frac{4}{7}\right)-P^{\prime}\left\{\frac{-8 k^{\prime} \zeta^{\prime}}{\Delta E\left(b_{1 \mathrm{~g}}\right)}-\frac{6 k \zeta}{7 \Delta E\left(e_{\mathrm{g}}\right)}\right. \\
& -\frac{4 k \zeta^{2}}{7\left[\Delta E\left(e_{\mathrm{g}}\right)\right]^{2}}-\frac{6 k \zeta^{\prime 2}}{7 \Delta E\left(e_{\mathrm{g}}\right) \Delta E\left(b_{1 \mathrm{~g}}\right)} \\
& \left.-\frac{22 k^{\prime} \zeta \zeta^{\prime}}{7 \Delta E\left(e_{\mathrm{g}}\right) \Delta E\left(b_{1 \mathrm{~g}}\right)}-\frac{6 g_{s} \zeta^{\prime 2}}{7\left[\Delta E\left(b_{1 \mathrm{~g}}\right)\right]^{2}}-\frac{17 g_{s} \zeta^{2}}{14\left[\Delta E\left(e_{\mathrm{g}}\right)\right]^{2}}\right\}, \\
& A_{\perp}=P\left\{\frac{2}{7}-\kappa-\frac{11 k \zeta}{7 \Delta E\left(e_{\mathrm{g}}\right)}+\frac{11 k \zeta^{2}}{14\left[\Delta E\left(e_{\mathrm{g}}\right)\right]^{2}}\right. \\
& \left.-\frac{11 g_{s} \zeta^{2}}{7\left[\Delta E\left(b_{1 \mathrm{~g}}\right)\right]^{2}}-\frac{11 g_{s} \zeta^{2}}{28\left[\Delta E\left(e_{\mathrm{g}}\right)\right]^{2}}\right\} .
\end{aligned}
$$

In Eqs. (3) and (4), $g_{s}(=2.0023)$ is the $g$ value of the free electron, $\kappa$ is the Fermi contact parameter; $k^{\prime}$ and $k$ are the orbital reduction factors; and the energy differences between excited states $b_{1 \mathrm{~g}}, e_{\mathrm{g}}$ and ground state $b_{2 \mathrm{~g}}$ are, respectively, $\Delta E\left(b_{1 \mathrm{~g}}\right)$ and $\Delta E\left(e_{\mathrm{g}}\right)$,

$$
\begin{aligned}
& \Delta E\left(b_{1 \mathrm{~g}}\right)=10 D q, \\
& \Delta E\left(e_{\mathrm{g}}\right)=-3 D s+5 D t .
\end{aligned}
$$

The two SO coupling parameters $\zeta$ and $\zeta^{\prime}$, two orbital reduction factors $k$ and $k^{\prime}$, and two dipole hyperfine structure parameters $P, P^{\prime}$ can be given as

$$
\begin{aligned}
& \zeta=N_{t}\left(\zeta_{d}^{0}+\lambda_{t}^{2} \zeta_{p}^{0} / 2\right), \\
& \zeta^{\prime}=\left(N_{t} N_{e}\right)^{1 / 2}\left(\zeta_{d}^{0}-\lambda_{t} \lambda_{e} \zeta_{p}^{0} / 2\right), \\
& k=N_{t}\left(1-2 \lambda_{t} S_{d p}\left(t_{2 \mathrm{~g}}\right)+\lambda_{t}^{2} / 2\right), \\
& k^{\prime}=\left(N_{t} N_{e}\right)^{1 / 2}\left(1-\lambda_{t} S_{d p}\left(t_{2 \mathrm{~g}}\right)-\lambda_{e} S_{d p}\left(e_{\mathrm{g}}\right)-\lambda_{t} \lambda_{e} / 2\right) \\
& P=N_{t} P_{0}, \quad P^{\prime}=\left(N_{t} N_{e}\right)^{1 / 2} P_{0},
\end{aligned}
$$

in which $N_{t}, N_{e}$ are the normalized parameters. $\lambda_{e}$, $\lambda_{t}$ are the orbital mixing coefficients, we assume that $\lambda_{\gamma} \approx \lambda_{e} \approx \lambda_{t}$ for the simplicity in the calculation. The group overlap integrals $S_{d p}\left(e_{\mathrm{g}}\right)$ and $S_{d p}\left(t_{2 \mathrm{~g}}\right)$ can be obtained from the Slater-type SCF functions [23, 24] and the metal-ligand reference distance $R_{0} . \quad P_{0}(=136 \times$ $\left.10^{-4} \mathrm{~cm}^{-1}[14,20]\right)$ is the hyperfine structure constant of the $\mathrm{V}^{4+}$ ion.

\section{Application}

From formulae (1)-(4), the relation between ESR spec$\operatorname{tra}\left(g_{\|}, g_{\perp}\right.$ and $\left.A_{\|}, A_{\perp}\right)$ and the local lattice structural parameters $\left(R_{\|}, R_{\perp}\right)$ has been established by using two SO coupling parameter model, the superposition model, and high-order perturbation theory. As an application, we calculated the ESR data and structural parameters of $\mathrm{V}^{4+}$ ion in ternary vanadate-lithium-borate glasses. The values of the fitting parameters can be obtained from the calculation of ESR data of $\mathrm{V}^{4+}$ in vanadate-lithiumborate glasses,

$$
\lambda_{\gamma} \approx \lambda_{e} \approx \lambda_{t} \approx 0.41, \quad R_{\|}=0.150 \mathrm{~nm}, \quad \kappa=0.81 .
$$

The correlative values of the overlap integrals, normalized parameters, two SO coupling parameters, and two orbital reduction factors are collected in Table I. The calculated ESR parameters are compared with the experimental values in Table II.

The correlative calculated parameters

TABLE I

for $\mathrm{V}^{4+}$ in vanadate-lithium-borate glasses.

\begin{tabular}{c|c|c|c|c|c|c|c}
\hline \hline$S_{d p}\left(t_{2 \mathrm{~g}}\right)$ & $S_{d p}\left(e_{\mathrm{g}}\right)$ & $N_{t}$ & $N_{e}$ & $\zeta$ & $\zeta^{\prime}$ & $k$ & $k^{\prime}$ \\
\hline 0.0508 & 0.1297 & 0.8877 & 0.9418 & 231.3 & 215.2 & 0.9254 & 0.7699
\end{tabular}

\section{Discussions and conclusions}

Majority vanadyl complexes [10, 14] and also oxovanadium coordination polyhedra realized in oxide glasses 
have practically the some pentacoordinated $C_{4 v}$ symmetry (square-pyramidal) where the $\mathrm{V}=\mathrm{O}$ bond oriented along the $\mathrm{C}_{4}$ axis is stronger [and $R(\mathrm{~V}=\mathrm{O})$ smaller] than the four $\mathrm{V}-\mathrm{O}$ bonds from $x O y$ plane (pyramidal base) $[10,14]$. In solutions and also in oxide glasses the coordination of the sixth oxygen atom below the $\mathrm{V}^{4+}$ site in opposition with "yl" oxygen forming $\mathrm{O}-\mathrm{VO}_{4}-\mathrm{O}$ units (hexacoordinated octahedral distorted) occur. In this case the $\mathrm{V}=\mathrm{O}$ bond weakens $(\mathrm{V}-\mathrm{O})$ and the $R_{\|}$increases [25-30]. Thus, vanadyl complexes $R_{\|}[\approx 1.5 \AA, \mathrm{R}(\mathrm{V}=\mathrm{O})$ bond] and $R_{\perp}[\approx 1.95 \AA, R(\mathrm{~V}-\mathrm{O})$ bonds] are suggested in present studied system.

In summary, from Table II, it is good agreement between the theoretical data and the experimental values of ESR spectra. It should be pointed out that the signs of hyperfine structure parameters $A_{\|}, A_{\perp}$ are actually given as absolute values in literature [9]. The negative signs of the calculated $A_{\|}, A_{\perp}$ are suggested in this work.

\section{TABLE II}

$g$ factors and hyperfine structure constants $A_{i}$ for $\mathrm{V}^{4+}$ in vanadate-lithium-borate glasses.

\begin{tabular}{c|c|c}
\hline \hline ESR parameters & Calc. & Expt. [9] \\
\hline$g_{\|}$ & 1.940 & $1.939(3)$ \\
$g_{\perp}$ & 1.983 & $1.998(3)$ \\
$A_{\|}\left[10^{-4} \mathrm{~cm}^{-1}\right]$ & -175 & $170.6-176.4^{a}$ \\
$A_{\perp}\left[10^{-4} \mathrm{~cm}^{-1}\right]$ & -65 & $61.3-71.4^{a}$ \\
\hline
\end{tabular}

${ }^{a}$ The data are given by absolute values.

The octahedron around $\mathrm{V}^{4+}$ ion is compressed along $\mathrm{C}_{4}$ axis because the calculated value of $R_{\|}$is less than that of $R_{\perp}$. The magnitude of the tetragonal distortion $\Delta R$ ( $=R_{\perp}-R_{\|}=0.045 \mathrm{~nm}$ ) has also been shown that $\mathrm{V}-\mathrm{O}$ bonds are stronger along the $\mathrm{C}_{4}$-axis direction than other $\mathrm{V}-\mathrm{O}$ bonds of the complex.

\section{Acknowledgments}

Project supported by the National Science Foundation of China (grant No. 11104366, 50876120), the Natural Science Foundation project of CQ (grant No. CSTC2011jjA50015, KJ120804 and KJ120826), the Key Project of Chinese Ministry of Education (No. 212139) and the Innovative Group of Science and Technology of Chongqing (grant No. 201023) and CQUT.

\section{References}

[1] S.P. Singh, R.P.S. Chakradhar, J.L. Rao, B. Karmakar, J. Alloys Comp. 493, 256 (2010).

[2] D.V.R. Murthy, B.C. Jamalaiah, T. Sasikala, L.R. Moorthy, M. Jayasimhadri, K. Jang, H.S. Lee, S.S. Yi, J.H. Jeong, Physica B 405, 1095 (2010).

[3] D. Prakash, V.P. Seth, I. Chand, P. Chand, J. Non- Cryst. Solids 204, 46 (1996).
[4] J.T. Gong, L.J. Wang, W.L. Feng, X.Z. Yang, F. Zhang, Acta Phys. Pol. A 120, 497 (2011).

[5] Q. Wei, Acta Phys. Pol. A 118, 670 (2010).

[6] V.P. Seth, A. Yadav, Phys. Chem. Glasses 28, 109 (1987).

[7] A. Yadav, V.P. Seth, S.K. Gupta, J. Non-Cryst. Solids 101, 1 (1988).

[8] A.A. Ahmed, A.F. Abbas, F.A. Moustafa, Phys. Chem. Glasses 24, 43 (1983).

[9] O. Cozar, I. Ardelean, V. Simon, G. Ilonca, C. Craciun, C. Cefan, J. Alloys Comp. 326, 124 (2001).

[10] D. Kivelson, S.K. Lee, J. Chem. Phys. 41, 1896 (1964).

[11] W.C. Zheng, Y.J. Fan, X.X. Wu, Z. Naturforsch. A 60, 433 (2005).

[12] M.L. Du, C. Rudowicz, Phys. Rev. B 46, 8974 (1992).

[13] W.L. Feng, T.X. Zeng, T.H. Chen, J.J. Chen, Cryst. Res. Technol. 45, 75 (2010).

[14] L.J. Boucher, E.C. Jynan, T.F. Yen, Electron Spin Resonance of Metal Complexes, Plenum Press, New York 1969

[15] D.J. Newman, B. Ng, Rep. Prog. Phys. 52, 699 (1989).

[16] Y.Y. Yeung, D.J. Newman, Phys. Rev. B 34, 2258 (1986).

[17] C. Rudowicz, Y.Y. Yeung, Z.Y. Yang, J. Qin, J. Phys., Condens. Matter 14, 5619 (2002).

[18] W.C. Zheng, Q. Zhou, Q, X.X. Wu, Y. Mei, Spectrochim. Acta A 61, 1243 (2005).

[19] W.L. Feng, Y.C. Zhong, F. Zhang, Q. Wei, X.H. Zhang, Philos. Mag. 90, 1213 (2010).

[20] R. Biyik, R. Tapramaz, B. Karabulut, Z. Naturforsch. A 58, 499 (2003).

[21] H.D. Bedon, W.E. Hatfield, S.M. Horner, S.Y. Tyree Jr, Inorg. Chem. 4, 743 (1965).

[22] J.S. Griffth, The Theory of Transition-Metal Ions, Cambridge University Press, London 1964.

[23] E. Clementi, D.L. Raimondi, J. Chem. Phys. 38, 2686 (1963).

[24] E. Clementi, D.L. Raimondi, W.P. Reinhardt, J. Chem. Phys. 47, 1300 (1976).

[25] H. Toyuki, S. Akagi, Phys. Chem. Glasses 13, 15 (1972).

[26] L.D. Bogomolova, V.A. Jachkin, V.N. Lazukin, T.K. Pavlushkina, V.A. Shmuckler, J. Non-Cryst. Solids 28, 375 (1978).

[27] H. Hosono, H. Kawazoe, T. Kanazawa, J. Non-Cryst. Solids 37, 427 (1980).

[28] A. Agarwal, A. Sheoran, S. Sanghi, V. Bhatnagar, S.K. Gupta, M. Arora, Spectrochim. Acta A 75,964 (2010).

[29] A. Yadav, V.P. Seth, J. Mater. Sci. 22, 239 (1987).

[30] S. Gupta, N. Khanijo, A. Mansingh, J. Non-Cryst. Solids 181, 58 (1995). 